\title{
Introduction to the Plasma State of Matter
}

\author{
B. R. Adhikari and *R. Khanal \\ Central Department of Physics, Tribhuvan University, Kirtipur, Nepal \\ Department of Physics, Bhaktapur Multiple Campus, Tribhuvan University, Bhaktapur, Nepal \\ *plasmanepal@hotmail.com
}

\begin{abstract}
Plasma is a quasineutral gas of charge and neutral particles which exhibits collective behavior. Because of its distinct behavior it is often termed as the fourth state of matter. It is of interest as it has found applications in diverse areas such as, controlled thermonuclear fusion, gas lasers, lightning, medicine, space science, electronics, etc. In this article we present an introduction to the plasma state of matter and discuss some of its applications.
\end{abstract}

Keywords: plasma, quasineutrality, collective behavior, Debye shielding, plasma frequency, controlled thermonuclear fusion

\section{INTRODUCTION}

As the temperature of any solid material is raised, its state changes from solid to liquid and then to gas. If we increase the temperature of a gas beyond a certain limit, it enters a regime where the thermal energy of its constituent particle is so great that the electrostatic forces which ordinarily bind electrons to atomic nuclei are overcome. Instead of hot gas composed of electrically neutral atoms, we then have a mixed population of charged and neutral particles. With increasing temperature the number of ionized particles increases and the ionized gas starts behaving differently. After the fraction of ionized particles is sufficiently high the ionized gas starts exhibiting the collective behavior and the state of matter is plasma, and it is neither solid nor liquid nor gas. Plasma is thus defined as a quasineutral gas of charge and neutral particles which exhibits collective behavior. Quasineutrality of plasma implies that the electron density $n_{e}$ is nearly equal to the ion density $n_{i}$ so that $n_{i} \approx n_{e} \approx n$, where $n$ is the common density of plasma particles called the plasma density; but the plasma is not so neutral that all the interesting electromagnetic phenomena vanish. The collective behavior implies that the motion of species depend not only on the local conditions but also on the state of the plasma far away from the point of interest [1]

The word "plasma" is derives from Greek and means something molded or fabricated. The existence of "the fourth state of matter" was first identified by
Sir William Crookes in 1879 , however, the term plasma was introduced by I. Langmuir in 1928 to describe the state of matter in the positive column of glow discharge tube [2]. Plasma is thus an ionized gas, satisfying certain criteria to be discussed in next Sections; however any ionized gas cannot be called plasma.

\section{DEBYE SHIELDING}

Plasma possesses a special ability to shield out external potentials that are applied to it within a very small region. The external potential $\left(\phi_{0}\right)$ applied to a plasma is distributed into the plasma as

$$
\phi(x)=\phi_{o} e^{-\frac{|x|}{\lambda_{D}}}
$$

where, $\lambda_{D}$ is the Debye length expressed as

$$
\lambda_{D}=\left(\frac{\varepsilon_{0} k T_{e}}{n e^{2}}\right)^{\frac{1}{2}}
$$

$\varepsilon_{0}$ is the permittivity of free space, $T_{e}$ is the electron temperature, $e$ is the electronic charge and $k$ is the Boltzmann constant.

The Debye length is an important physical property for the description of plasma. It provides a measure of the distance over which the influence of external potential is felt by the other charged particles inside the plasma. From equation (1) it is obvious that the charged particles arrange themselves in such a way that the external electrostatic fields are shielded within a distance of the order of a few Debye lengths. 
This property is called the Debye shielding and for plasma to exhibit the collective behavior the Debye length must be much smaller than its characteristic length so that the majority of plasma particle are not influenced by the external potential. Consequently, charge in the plasma interacts collectively only with the charges those lie inside its Debye sphere, its effect on the other charges being effectively negligible.

For the Debye shielding to be a statistically valid concept the number of particles inside a Debye sphere must be sufficiently large [3], i.e.,

$$
N_{D}=\frac{4}{3} \frac{\pi}{n^{1 / 2}}\left(\frac{\varepsilon_{0} k T_{e}}{e^{2}}\right)^{\frac{3}{2}}
$$

must be very large.

\section{PLASMA OSCILLATION}

If the electrons in a quasineutral plasma are displaced from its equilibrium position an electric field will be built in such a direction such that it will try to restore the neutrality of the plasma by pulling the electrons back to their original positions. As the ions are massive compared to the electrons they form a uniform background. Due to inertia, the electrons will overshoot and oscillate about their equilibrium positions with a characteristic frequency, which is known as plasma frequency. This oscillation is so fast that the massive ions do not have time to respond to the oscillating electric field and may be considered as fixed. On solving the basic fluid equations for a plasma with singly charged ions the electron-plasma frequency $\omega_{p}$ is [1]

$$
\omega_{p}=\left(\frac{n e^{2}}{\varepsilon_{0} m_{e}}\right)^{2}
$$

where $m_{e}$ stands for mass of an electron. For the properties of the plasma to be determined by electromagnetic rather than hydrodynamic collision the plasma frequency must be large compared to the ordinary collision frequency.

\section{CRITERION FOR PLASMA}

As already mentioned, all the plasma are ionized gases, however, not all ionized gases are plasma. The conditions an ionized gas must satisfy to be called plasma are:

i) $\lambda_{D}<L$, where $L$ is the dimension of plasma in question.

ii) $N_{D}>>>1$

iii) $\omega_{p} \omega_{c}>1$, where $\omega_{c}$ is the collision frequency of electrons with neutral

It is often said that more than $99 \%$ of the matter in the visible universe is in plasma state, however, the state of matter is almost not present on earth. A rare example of naturally occurring high temperature plasma on earth is lightning. This type of plasma can also be generated artificially using a high voltage, high temperature arc, which is the basis for the corona discharge process and for the plasma torch used to vaporize and redeposit metals. As soon as we start moving outside from the earth surface, e.g., the ionosphere is dominated by plasma. Although the plasma state is very rare on earth it is interesting to note that our solar system is dominated by plasma, as the Sun is in plasma state. Other examples where plasmas can be found are in stars, lightening, solar wind, fusion devices, welding arcs, neon signs, aurora, nebulae, galaxies, etc.

It is often a misconception that plasma is always hot. It is interesting to note that plasmas can exist in diverse ranges of temperature and density as shown in Fig. 1. Low temperature plasmas, used in surface modification and organic cleaning, are ionized gases generated at pressures between 0.1 and 2 Torr [4]. Thus plasma can exist at density and temperature both having ranges much lower as well as much higher than that of solid, liquid, and gaseous states of matter. Because of this diverse nature it has found applications in various fields, some of which are discussed below. 


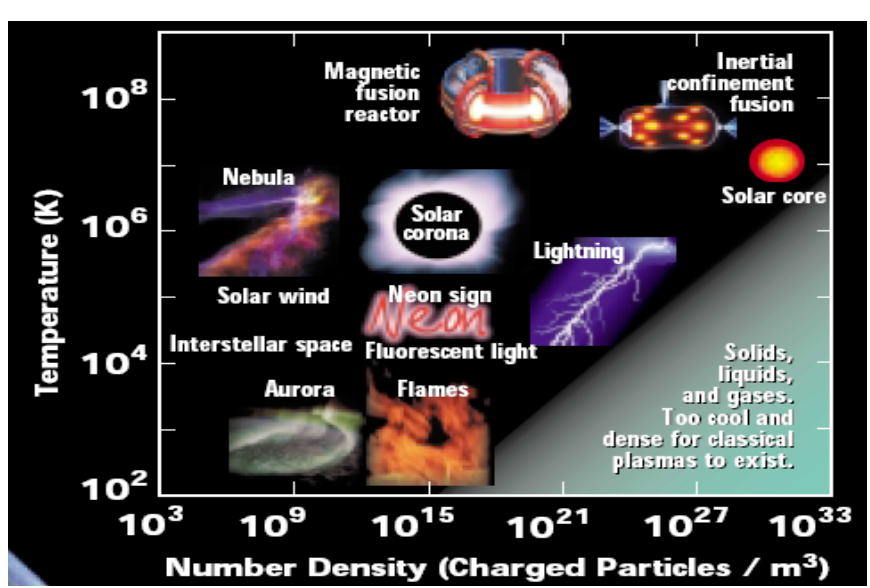

Fig. 1: Existence of plasma in diverse ranges of temperature and density

\section{APPLICATIONS OF PLASMA}

Because of its diverse nature plasma finds application in various fields such controlled thermonuclear fusion, surface treatment, biomedical applications, lighting, medicine, electronics, transportation and space propulsion, display technology, space physics, solid state plasma, gas lasers, etc. Some of the important and interesting applications of plasma in various fields are discussed here.

\section{CONTROLLED THERMONUCLEAR FUSION:}

Nuclear fusion as the source of energy of the Sun and stars was suggested many years ago [5-7]. Since then, fusion energy is considered as one of the best potential sources of virtually unlimited energy for mankind. Since nuclear fusion can potentially provide and almost unlimited source of energy, it is one of the most important scientific challenges man faces today. The most suitable reaction for the best utilization of the fusion energy as the potential sources of virtually unlimited energy for mankind is the one involving deuterium (D) and tritium (T) as:

$$
\mathrm{D}+\mathrm{T}{ }^{\circledR}{ }^{4} \mathrm{He}+\mathrm{n}+17.6 \mathrm{MeV}
$$

The Lawson criterion for a useful nuclear fusion reaction suggests that the cross-sections for the fusion reaction is appreciable for very high incident energies which corresponds to the temperature of the order of $10^{8} \mathrm{~K}$. This is much higher than the temperature at the core of the Sun and hence it is not easy confining the fusion fuels. This is in fact the problem faced by scientists which is not yet fully resolved and hence the fusion power could not be produced for commercial uses, so far. On the other hand the problem of heating and containing such plasma is responsible for the rapid growth of plasma physics since 1950s.

The schemes for realization of fusion energy on earth can be categorized into two main schemes, namely Magnetic Confinement Fusion (MCF) and Inertial Confinement Fusion (ICF) [8-10]. In $\mathrm{MCF}$, the plasma is confined using a sophisticated nested magnetic field lines, where as in ICF, high power lasers (and in some cases high power particle beams) are used to compress small pellets of plasma fuel to very high densities and temperature within a time of the order of nanoseconds. Among various magnetic confinement schemes the most successful device so far is 'Tokamak', a doughnut shaped magnetic chamber initiated in former Soviet Union in early 50s. Based on various experiments the next generation Tokamak named "International Thermonuclear Experimental Reactor (ITER)", an international nuclear fusion research project to built the largest and most advanced experimental Takamak ever built, is currently under construction at Cadarache in France. The first operation of ITER is expected to be in 2020 [11].

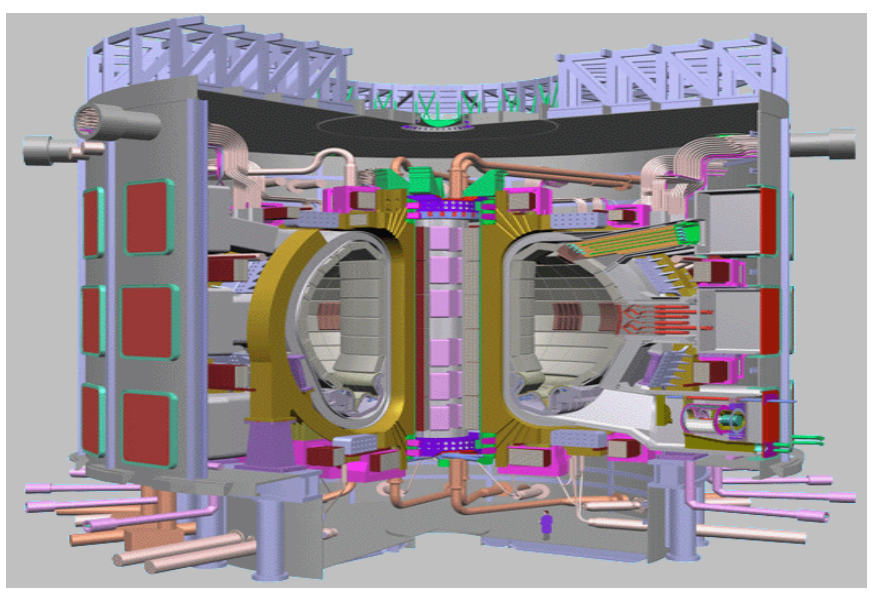

Fig. 2: Artist's impression of the International Thermonuclear Experimental Reactor (ITER)

\section{PLASMA SURFACE TREATMENT:}

Various plasma processes have been developed to attain a variety of specific surface properties and here we discuss some of the applications that are 
also being studied in the plasma research lab at Kathmandu University, Dhulikhel, Nepal.

\subsection{Surface Cleaning:}

Plasma treatment is a safe and environmentally friendly alternative to traditional cleaning methods. A wide variety of industries utilize gas plasma treatment to remove organic surface contamination from materials that require critical cleaning. The active species in the oxygen combined with UV energy creates a chemical reaction with the surface contaminants, resulting in their volatilization and removal from the reaction chamber. Applications include cleaning of circuit boards for improved wire bonding, removal of organic contamination from glass slides and flat panels, cleaning of medical devices and other materials such as various metals and ceramics.

\subsection{Adhesion promotion:}

Improving adhesion between two surfaces is a common application. Good adhesion requires strong interfacial forces via chemical compatibility and chemical bonding. Plasma surface treatment can also assist in creating chemically active functional groups, such as amine, carbonyl, hydroxyl and carboxyl groups, to improve interfacial adhesion. Common applications include pre-treatment for medical catheters, cardiovascular stents, syringe components, dialysis pump parts and plastic films for drug bags. Plasma is also used to improve bond ability on substrates, such as glass, polymers, ceramics and various metals.

\subsection{Controlling surface energies:}

Plasma processing can also be used to tailor surface energies. Hydrophilic and hydrophobic surfaces can be created on polymers through interaction with a gas plasma. Using oxygen to create hydroxil functionality increases the wettability of a surface. This process has been used to enhance the performance of a catheter by creating a wettable surface on the polymer tubing. Similarly, surfaces can be specifically engineered to modify protein binding and improve blood compatibility. Common applications include tissue culture wares, filtration or separation media and contact lenses.

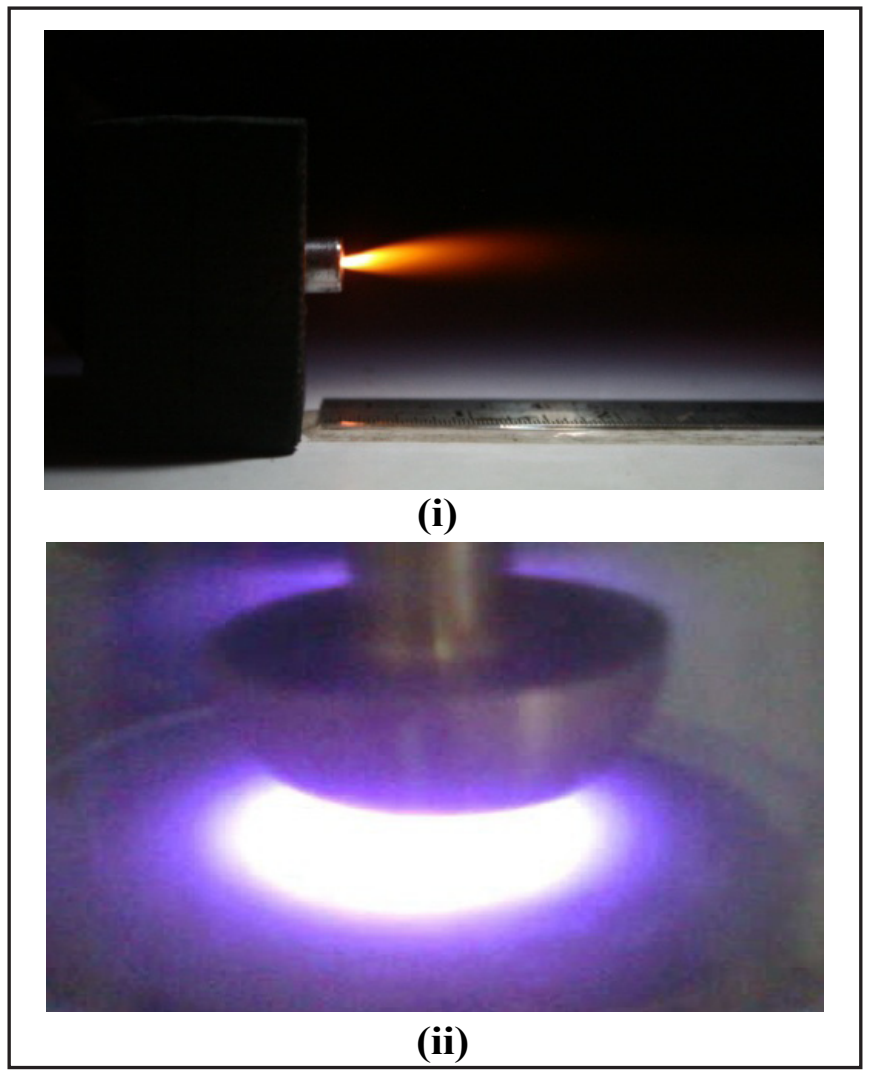

Fig. 3: (i) Atmospheric Pressure Plasma Jet and (ii) Dielectric Barrier Discharge (DBD) in Nitrogen

\subsection{Improving biocompatibility:}

Biomaterials that come in contact with blood or protein require special surface treatments to enhance biocompatibility. Amine functional groups, which are attached by ammonia plasma treatment, act as hooks for anticoagulants, such as heparin, and thereby decrease thrombogenicity. Synthetic polymer implant materials can be surface activated using radio frequency plasma techniques to enable covalent immobilization of cell binding peptides derived from the extra-cellular matrix proteins: fibronectin and laminin. The resulting grafted peptides can promote complete coverage of a surface with a monolayer of intact, healthy endothelial cells to form a natural, blood compatible surface.

\subsection{Enhancing performance:}

Surface crosslinking is often used to enhance the performance of polymers. The activity of the plasma creates a higher crosslinking density within the material to depths of a few thousand angstroms. The 
resulting increase in hardness and chemical resistance can enhance performance in many applications.

\section{PLASMA LIGHTING}

The most prevalent man-made plasmas on our planet are the plasmas in lamps. There are primarily two types of plasma-based light sources, fluorescent lamps and high-intensity arc lamps. Fluorescent lamps find widespread use in homes, industry and commercial settings. High-intensity sources are widely used in industrial and commercial settings as well as for outdoor and security lighting near homes and public areas. Inside every fluorescent lamp there lurks plasma. It is the plasma that converts electrical power to a form that causes the lamp's phosphor coating to produce the light we see. The phosphor is the white coating on the lamp wall. A fluorescent lamp is shown here with part of the phosphor coating removed to reveal the blue plasma glow inside. The plasma generates ultraviolet light which in turn excites the phosphor coating inside the glass envelope. The phosphor emits a single color of visible light. Each pixel consists of three sub-pixels, one each of red, green and blue. By combining these primary colors at varying intensities, all colors can be formed.
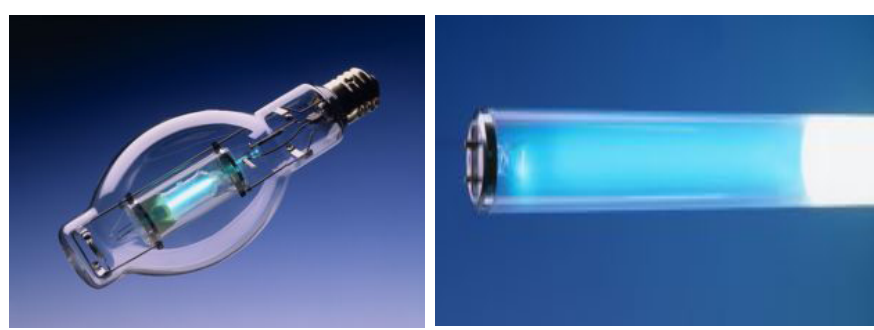

Fig. 3: Plasma lightning

\section{BIOMEDICAL APPLICATIONS:}

Although many synthetic biomaterials have physical properties that meet and even exceed those of natural body tissue, they can often cause adverse physiological reactions such as infection, inflammation and thrombosis formation. Through surface modification, biocompatibility as well as biofunctionality can be achieved without changing the bulk properties of the material. There are many ways by which to alter the interaction of biomaterials with their physiological environments, of these, plasma surface modification provides device manufacturers with a flexible, safe and environmentally friendly process that is extremely effective. Plasma surface modification is used for a variety of biomedical applications like Bioseparation, Sterilization, Ocular prostheses, Orthopaedic applications, Tissue culturing, etc.

\section{CONCLUSION}

The materials in our immediate surroundings are mostly solid, liquid and gas; however; the plasma state of matter dominates in our universe. It is the stuff in our sun and other stars and of the vast interstellar space. Plasma has found applications in lightning, computer chip manufacturing, spaceship propulsion, and many more. In the future it will likely be the stuff of electric power generated from fusion. Nuclear Fusion provides one of the major options for the truly sustainable or long term energy source. The fusion fuels are virtually inexhaustible and readily available throughout the world. Fusion power plant operation is inherently safe and has no risk of long-lived radioactive waste. Realizing these reactions to produce energy on earth is still a grand challenge to scientists and engineers. Though a steady progress has been made still several scientific and technological advances are necessary before the dream of commercial electricity production will become a reality.

\section{REFERENCES}

[1] A. S. Eddington, The Internal Constitution of the Stars, Cambridge University Press (1926)

[2] F. F. Chen. Introduction to Plasma Physics and Controlled Fusion (Second edition), Plenum Press, New York (1984)

[3] H. A. Bethe and C. L. Crictchfield, Phys. Rev. 54, 248 (1938)

[4] H. A. Bethe, Phys. Rev. 55, 434 (1939)

[5] I. Langmuir, Phys. Rev. 23, 954 (1929)

[6] J.A. Bittencourt, Fundamentals of Plasma Physics (Third edition), Springer, New York (2004)

[7] J. Nuckolls et al, Nature 239, 139 (1972)

[8] N. G. Basov and O. N. Krokhin, Sov. Phys.JETP 19, 123 (1964)

[9] V. B. Rozanov et al, Energy from Inertial Fusion, IAEA, Vienna, (1995)

[10] www.plasmas. org

[11] www.iter.org 